\title{
Stromal upregulation of lateral epithelial adhesions: Gene expression analysis of signalling pathways in prostate epithelium
}

Karen F Chambers ${ }^{1}$, Joanna F Pearson ${ }^{1}$, Davide Pellacani ${ }^{1}$, Naveed Aziz ${ }^{2}$, Miodrag Gužvić ${ }^{3}$, Christoph A Klein ${ }^{3}$ and Shona H Lang ${ }^{1 *}$

\begin{abstract}
Background: Stromal signalling increases the lateral cell adhesions of prostate epithelial cells grown in 3D culture. The aim of this study was to use microarray analysis to identify significant epithelial signalling pathways and genes in this process.

Methods: Microarray analysis was used to identify genes that were differentially expressed when epithelial cells were grown in 3D Matrigel culture with stromal co-culture compared to without stroma. Two culture models were employed: primary epithelial cells (ten samples) and an epithelial cell line (three experiments). A separate microarray analysis was performed on each model system and then compared to identify tissue-relevant genes in a cell line model.

Results: TGF beta signalling was significantly ranked for both model systems and in both models the TGF beta signalling gene SOX4 was significantly down regulated. Analysis of all differentially expressed genes to identify genes that were common to both models found several morphology related gene clusters; actin binding (DIAPH2, FHOD3, ABLIM1, TMOD4, MYH10), GTPase activator activity (BCR, MYH10), cytoskeleton (MAP2, MYH10, TMOD4, FHOD3), protein binding (ITGA6, CD44), proteinaceous extracellular matrix (NID2, CILP2), ion channel/ ion transporter activity (CACNA1C, CACNB2, KCNH2, SLC8A1, SLC39A9) and genes associated with developmental pathways (POFUT1, FZD2, HOXA5, IRX2, FGF11, SOX4, SMARCC1).

Conclusions: In 3D prostate cultures, stromal cells increase lateral epithelial cell adhesions. We show that this morphological effect is associated with gene expression changes to TGF beta signalling, cytoskeleton and anion activity.
\end{abstract}

\section{Background}

Tissue morphogenesis is controlled by a variety of factors including local growth factors, extracellular matrix, cell adhesion molecules and the cytoskeleton. Cadherins and tight junctions have a major role in establishing and maintaining intercellular adhesion [1,2]. E-cadherin initiates intercellular contacts, forms homophilic adhesions and links to the actin cytoskeleton through $\beta$-catenin. The spatial control of cadherin clusters by the actin cytoskeleton is important for stable adhesions [3,4]. In

\footnotetext{
* Correspondence: lang.short@talktalk.net

'Yorkshire Cancer Research Unit, Dept. Biology, University of York, Heslington, York, (YO10 5YW), UK

Full list of author information is available at the end of the article
}

adult polarised epithelial tissues adherens junctions are further associated with tight junctions leading to the formation of the apical junctional complex. Tight junctions provide epithelial cells with a paracellular diffusion barrier that is critical for normal tissue function and maintenance of polarity $[5,6]$. The shape of an epithelial cell is related to its function, to adhesion molecules and to their interaction with an organised actin cytoskeleton. The mechanisms controlling lateral cell adhesions in an adult tissue are not fully understood. An understanding of the molecular pathways which govern junctional proteins and actin cytoskeleton organization are required to further our understanding of normal tissue and the development of diseases.

\section{C) Biomed Central}


We have previously modelled prostate epithelial morphogenesis using 3D Matrigel culture [7]. Primary epithelial cells, grown in 3D Matrigel, form hollow acinus-like gland structures and co-culture of these structures with stromal cells leads to increased polarisation and increased lateral cell adhesions between the epithelial cells. Significantly, this result contradicts the role of stroma in epithelial mesenchymal transition [8] and suggests that the role of stroma in 3D culture supports a role for stroma in the maintenance of tissue integrity. In support of this, mouse modelling of the prostate also demonstrated the requirement for stroma to induce architectural organisation [9]. Our recent work has demonstrated that stromal derived TGF $\beta 2$ can increase the co-localisation of E-cadherin with the actin cytoskeleton and decrease paracellular permeability (paper in submission). The control of any biological process is highly complex, involving many signalling pathways. To identify epithelial genes and signalling pathways that are controlled by stromal cells in 3D culture, we employed microarray analysis and bioinformatics. Micorarray information derived from limited numbers of cell lines does not always represent information derived from tissue. However cell lines provide useful reproducible model systems in the laboratory with which to understand complex biological processes. Therefore we aimed to combine microarray information derived from both primary and cell line cultures, to identify genes that are relevant to tissues, but could be further investigated in cell line model systems. Key pathways and gene clusters were identified that were associated with TGF beta signalling, cytoskeleton, ion channel/ion transporter activity and developmental pathways.

\section{Methods}

\section{Primary culture}

The use of human prostate tissue to grow primary cultures and patient consent procedures were approved by York Research Ethics Committee, (YREC Reference 91/ 7/6) and Hull and East Riding Local Research Ethics Committee (REC Reference Number 07/H1304/121). Tissue was obtained from York District Hospital, York and Castle Hill Hospital, Hull, UK. All patients who provided tissue gave their written consent. Tissues were given a unique identification number which was stored with the consent forms at participating hospitals, whilst documentation of tissue processing, experimentation and storage occurred at the YCR Cancer Research Laboratory.

Primary cultures were prepared as described before [7]. Briefly, prostatic tissue was digested with collagenase and trypsin, and differential centrifugation was used to enrich for epithelial and stromal fractions. The enriched stromal fraction was resuspended in stromal cell growth medium (RPMI1640 supplemented with10\% FCS and $1 \%$ antibiotic/antimycotic solution) and cultured routinely in $75-\mathrm{ml}$ tissue culture flasks. The epithelial fraction was resuspended in keratinocyte serum-free medium (KSFM) supplemented with $5 \mathrm{ng} / \mathrm{ml}$ epidermal growth factor and $1 \%$ antibiotic/antimycotic solution (medium subsequently referred to as KSFM.

\section{D Matrigel culture}

BPH-1 cells (benign prostate cell line), primary human benign prostate epithelial cultures and primary human benign prostate stromal cultures were cultured in 3D as described previously $[7,10]$.

Briefly, Primary stromal cultures (passage 1 to 3 ) were seeded prior to co-culture in $0.4 \mu \mathrm{m}$ Millicell-PCF inserts (Millipore, Livingston, UK), $2 \times 10^{4}$ cells/insert in RPMI supplemented with $10 \%$ FCS, until confluent. Epithelial cells were seeded at 5000 cells/ml in KE2 (KSFM, 2\% FCS, 2 mM L-glutamine, $10 \mathrm{nM}$ dihydrotestosterone and $10 \mathrm{nM} \beta$-estradiol) and 4\% (v/v) Matrigel. Inserts were then washed with PBS and added to epithelia plus Matrigel or blank wells, with KE2. The inserts were replaced 4, 8 and 12 days after cell seeding with fresh inserts of pre-seeded stroma. Medium was replenished at the same time through the removal of $0.5 \mathrm{ml}$ spent media and the addition of $0.5 \mathrm{ml}$ fresh KE2 supplemented with $4 \%$ Matrigel. Spheroids for RT-PCR were isolated from the Matrigel using BD Cell recovery solution (Becton Dickinson, Plymouth, UK).

mRNA isolation, cDNA synthesis and global amplification from a single spheroid for Operon array

Ten primary epithelial cultures (passage 1) were grown in Matrigel, with or without primary prostate stroma for 14 days, the optimum time of primary spheroid formation [7]. Single acini were isolated by "picking" spheroids with a pipette from a PBS/Matrgel suspension on a blocked dish $(0.3 \%$ BSA). RNA was prepared from the single spheroid amplification step according to a previous method [11].

\section{Operon array analysis}

Array pre-processing and significance analysis was performed using GeneSpring GX 10 software (Agilent Technologies, Inc). Arrays were filtered on expression between the 20th and 100th percentile of the raw data. Normalization was performed by scaling and baseline transformation to the median of all samples. The experiment was analysed as a reference design. Differentially expressed genes were identified by using a paired t-test with asymptotic p-value computation and no multiple testing correction where significance level was set at $\mathrm{p}>$ 0.05 . Genes that were $>1.1$ fold up- or down-regulated between groups were selected, this was then referred to 
as the 'primary 1.1 fold gene list. [Accession number; EMEXP-2994].

\section{Affymetrix microarray and analysis}

Three replicate cultures of BPH-1 (passage 47) were grown in 24 well plates with or without stroma (a mix of 3 different stromal cultures at passage 2) for 7 days in KE2 media. A time point of 7 days was chosen, since $\mathrm{BPH}-1$ cells grow faster than primary cultures. On average, 7 day old $\mathrm{BPH}-1$ acini are the same size as 14 day old primary acini. 3D acini produced by BPH-1 cells are predominantly homogeneous, therefore individual acini were not isolated, RNA was prepared from whole cultures and an Affymetrix array was performed. RNA was prepared using Illustra RNA Spin mini kit (GE Healthcare, Little Chalfont, UK). RNA samples were analysed using Affymetrix Human Genome U133 Plus 2.0 chips (Affymetrix Inc., Santa Clara, CA). Each array contains more than 54,676 probe sets that represent more than 47,000 transcripts. The RNA hybridisation of all Affymetrix U133 Plus 2.0 arrays was performed at TF facility (University of York). The cRNA synthesis of the samples was carried out according to the manufacturer's protocol. The fluorescence intensity for each chip was captured with an (Affymetrix GeneChip Scanner 3000). Affymetrix Microarray Suite version 5.0 (MAS 5.0, Affymetrix) was used to quantitate each chip. The raw data (CEL) files, were loaded into the DNA-chip analyser software (dChip) version Feb 2009 [12]. Normalisation was carried out using Invariant Set Normalisation method and probe expression values were then calculated using the perfect match (PM)-only model according to Chambers et al., 2009 [13]. Unsupervised hierarchical sample clustering was performed on a list of probes derived by filtering probes using the criteria of standard deviation divided by the mean between 0 and 1000 across the samples and the samples clustered into two separate groups, indicating reproducibility of the data. Three comparison criteria were applied to the data to detect differentially expressed genes by model based expression: 1) the fold change between the group means was chosen to exceed 1.5 fold 2) absolute difference between the two groups means $>50$ to eliminate the very low expressing genes that have intensity close to background levels and 3) a p-value of 0.05 for Welch's modified 2-sample paired t-test, adjusted to compensate for multiple testing using False discovery rate (FDR). In dChip, the FDR was estimated by a 1000 permutations.

Raw data was processed using the Affymetrix GCOS 1.2 software. After hybridization and scanning, probe cell intensities were calculated and summarized for the respective probe sets by means of the MAS5 algorithm. To compare the expression values of the genes from chip to chip, global scaling was performed, which resulted in the normalization of the trimmed mean of each chip to target intensity (TGT value) of 500 as detailed in the statistical algorithms description document of Affymetrix (2002; http://www.affymetrix.com). Each sample and hybridization underwent a quality control evaluation mainly checking for adequate scaling factors (1-3 for all samples), percentage of probe sets reliably detected (between $40-60 \%$ present call), and optimal 3'/5' hybridization ratios $(\sim 1)$ for the housekeeping genes (e.g., GAPDH), poly(A) spike-in controls, and the prokaryotic controls (bioB, bioC, bioD and cre). MAS5 normalised data were collected and analyzed using the GeneSpring GX10 Expression software (Agilent Technologies, USA). Differentially expressed genes were identified by using a two-class $t$ test where significance level was set at $\mathrm{p}>0.05$. Genes that were $>1.1$ fold up- or down-regulated between groups were selected. [Accession number; E-MEXP-2990].

\section{Pathway Express}

Functional analysis was performed on the 1.1, p $<0.05$ probe lists using Pathway-Express http://vortex.cs.wayne. edu[14]. Pathway Express ranks pathways using classical impact factors but deepens the statistics by adding into the analysis, the magnitude of gene expression change and the position and interaction within the pathway. The gamma p-value is provided by the impact analysis.

\section{Analysis of common genes from the primary culture and cell line arrays}

Two separate fold change lists were generated using Genesping. Both lists were generated using the same fold change of 1.1 fold and a $p<0.05$. The first list describes all the probes changing in $\mathrm{BPH}-1$ acini cultured with stroma compared to control $\mathrm{BPH}-1$ spheroids cultured without stroma. The second list describes the probes changing from single primary prostate acini cultured with and without stroma. To compare the genes that were the common between both lists and thus compare the BPH-1 cell line acini to primary acini the Entrez gene IDs were used. The probe IDs could not be used as Operon arrays were used for the single primary acini culture and Affymetrix arrays were used for the whole population $\mathrm{BPH}-1$ cell culture. The up-regulated and down-regulated Entrez IDs between the two lists were compared using the following function in excel VLOOKUP(B3,A:A,1,FALSE). The Entrez IDs that matched were copied into a txt. file and the gene names found using http://david.abcc.ncifcrf.gov/conversion.jsp.

\section{RT2 Profiler PCR Array}

RNA was prepared from BPH-1 spheroids grown in 24 well plates using Illustra RNA Spin mini kit (GE Healthcare, Little Chalfont, UK) and grown with and without 
stroma (a mix of 3 different stromal cultures at passage 2 , different cultures to the array). Reverse transcription was performed with RT2 PCR array First Strand Kit (SABiosciences). RT2 profiler PCR array for the human TGFB BMP signaling pathway were prepared as per manufacturers protocol. Target cDNA levels were detected using the ABI prism 7300 sequence detection system (Applied Biosciences) and normalised to HPRT, B2M, RPL13A and ACTB using the DDCt Data analysis method. The real time PCR conditions were as follows: 1 cycle at $95^{\circ} \mathrm{C}$ for $10 \mathrm{~min}, 40$ cycles at $95^{\circ} \mathrm{C}$ for $15 \mathrm{~s}$, and $60^{\circ} \mathrm{C}$ for $1 \mathrm{~min}$. 49 genes appearing on the TGF beta PCR array were not differentially expressed according to the microarray data.

\section{Real-time quantitative PCR (QRT-PCR)}

RNA was prepared from spheroids using Illustra RNA Spin mini kit (GE Healthcare, Little Chalfont, UK). Reverse transcription was performed with random hexamers (SuperScript ${ }^{\mathrm{TM}}$ II Reverse Transcriptase kit, Invitrogen). Quantitative real time PCR oligonucleotide primers (Additional file 1, Table S1) and fluorigenic Taqman probes were designed using Primer Express 3.0 software (ABI Prism; Applied Biosystems). Reactions used Taqman one-step mastermix kit (Applied biosystems), $400 \mathrm{nM}$ of each gene specific primer, $100 \mathrm{nM}$ each probe and $100 \mathrm{ng}$ total cDNA ( $25 \mu \mathrm{l}$ volume). Target mRNA levels were detected using the ABI prism 7700 sequence detection system (Applied Biosciences) and normalised to HPRT using the relative quantification method. The real time PCR conditions were as follows: 1 cycle at $50^{\circ} \mathrm{C}$ for $2 \mathrm{~min}, 1$ cycle at $95^{\circ} \mathrm{C}$ for 10 min, 40 cycles at $95^{\circ} \mathrm{C}$ for $15 \mathrm{~s}$, and $60^{\circ} \mathrm{C}$ for $1 \mathrm{~min}$. Assays consisted of three technical replicates.

\section{Results}

Primary epithelial gene expression changes and pathways induced by stromal secreted factors in 3D culture

To identify the pathways and genes that control lateral epithelial adhesions in prostate cells we compared the RNA expression patterns between 3D acini grown with and without stromal co-culture in 3D. To identify tissue relevant genes and not just gene changes associated with a single cell line we chose to perform our experiments on primary epithelial and primary stromal cell cultures. Most primary epithelial cells grown in 3D gels develop into clusters of spherical-acinus-like structures however some cells undergo limited divisions and others do not divide at all. To avoid immature acini or single cells contaminating our results we isolated individual acini (ten primary epithelial cell cultures) and hybridized their transcriptomes on an Operon microarray, which is known to be robust for low cell numbers [11]. Comparison of RNA expression patterns from 3D acini cultured with and without stroma identified 1574 probe sets with significant differential expression in between the two groups (paired t test, $\mathrm{p}<0.05$ ). We used principal component analysis to demonstrate that the genes identified could be used to distinguish between stromal co-culture or not within our data set (Figure 1). The ten most upregulated and down regulated genes are listed in Table 1 . To identify key functional categories within the differentially expressed genes we performed Pathway Express analysis (Table 2). Thirteen pathways were significantly ranked. The most highly ranked pathway was cell adhesion molecules, which predominantly indicated the upregulation and down regulation of claudin (CLDN) and integrin (ITG) isoforms.

\section{Heterogeneity in gene expression between primary epithelial cell samples}

To verify the Operon microarray data we selected FGFBP1 (fibroblast growth factor binding protein 1), since the average expression of this genes was highly upregulated in the presence of stroma. Using matched patients samples to the microarray, we performed quantitative RT-PCR (QRT-PCR). QRT-PCR confirmed the upregulation of FGFBP1 in six primary epithelial samples in response to stromal co-culture (Figure 2A). One epithelial sample showed no change in gene expression by array data but upregulation by QRT-PCR. Three samples showed down-regulation from the array data, but insufficient material prevented QRT-PCR analysis. Therefore, we observed good confirmation of the microarray analysis by QRT-PCR, but analysis of individual patient data sets indicated that different epithelial cultures had very variable expression of FGFBP1. Further

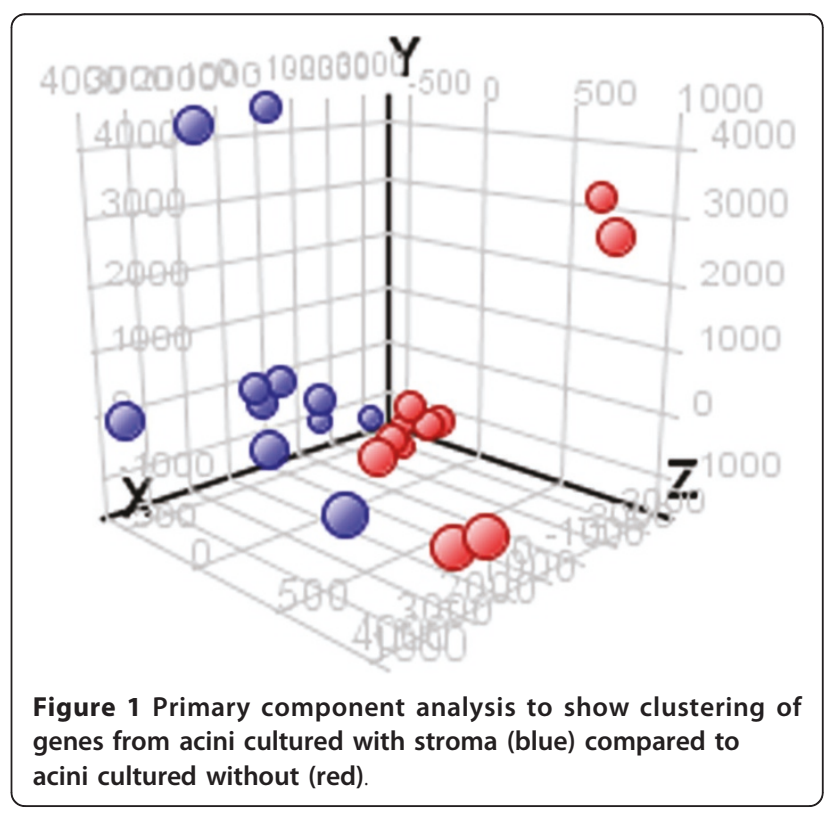


Table 1 The ten most upregulated and down regulated primary epithelial genes in response to stroma, in 3D culture

\begin{tabular}{|c|c|c|c|}
\hline Gene Symbol & Gene Title & Fold change & Probe ID \\
\hline FGFBP1 & fibroblast growth factor binding protein 1 & 3.078 & H300006842 \\
\hline OR4N5 & olfactory receptor, family 4 , subfamily $N$, member 5 & 2.993 & opHsV0400002653 \\
\hline KLRC4 & killer cell lectin-like receptor subfamily $C$, member 4 & 2.735 & H300021805 \\
\hline TESSP1 & testis serine protease 1 & 2.656 & opHsV0400002778 \\
\hline KT3K & Ketosamine-3-kinase & 2.528 & H200004088 \\
\hline KLRC1 & killer cell lectin-like receptor subfamily C, member 1 & 2.420 & H200005914 \\
\hline SNX12 & sorting nexin 12 & 2.330 & H200009199 \\
\hline PABPC1 & poly(A) binding protein, cytoplasmic 1 & 2.323 & H200014179 \\
\hline DNMBP & Dynamin binding protein & 2.282 & opHsV0400003719 \\
\hline MSH4 & mutS homolog 4 & 2.265 & H200011946 \\
\hline IL23R & interleukin 23 receptor & -2.489 & H300020818 \\
\hline DYNC2LI1 & dynein 2 light intermediate chain isoform 1 & -2.553 & opHsV0400000277 \\
\hline KRT6B & keratin $6 \mathrm{~B}$ & -2.560 & H300014335 \\
\hline SAA1 & serum amyloid $A 1$ & -2.600 & opHsV0400005893 \\
\hline TOP1 & topoisomerase (DNA) I & -2.844 & opHsV0400006965 \\
\hline PTPLAD1 & protein tyrosine phosphatase domain containing 1 & -2.859 & H300003447 \\
\hline SEMA3C & sema domain, secreted $3 C$ & -3.412 & H200008006 \\
\hline KRT23 & keratin 23 & -3.552 & H300014341 \\
\hline $\mathrm{C} 3$ & complement component 3 & -3.553 & $\mathrm{H} 20000880$ \\
\hline MAGI1 & membrane associated guanylate kinase, WWW and PDZ domain containing 1 & -4.678 & H200002533 \\
\hline
\end{tabular}

Values are the mean fold expression changes of ten primary epithelial cell cultures, abstracted from Operon datasets. All genes showed significant differential expression $(p<0.05)$. Positive values indicate up regulation, negative values indicate down regulation of epithelial genes cultured with stroma compared to without stroma. Hypothetical or unassigned genes were removed from the list.

Table 2 Significantly induced pathways associated with the response of primary epithelia to stroma co-culture in 3D

\begin{tabular}{|c|c|c|c|c|c|}
\hline Rank & Pathway & $\begin{array}{l}\text { Impact } \\
\text { Factor }\end{array}$ & $\begin{array}{l}\text { no. genes/ genes in } \\
\text { pathway }\end{array}$ & Upregulated genes & Downregulated genes \\
\hline 1 & Cell adhesion molecules & 246.278 & $9 / 133$ & $\begin{array}{c}\text { CLDN22, CLDN6, HLA-DPB1, ITGB2, NRXN3, } \\
\text { PTPRC }\end{array}$ & CD22, CLDN7, ITGA6 \\
\hline 2 & $\begin{array}{l}\text { Phosphatidyl inositol } \\
\text { signalling }\end{array}$ & 27.591 & $4 / 77$ & PRKCB1 & DGKG, INPP4B, PLCD3 \\
\hline 3 & $\begin{array}{l}\text { Antigen processing and } \\
\text { presentation }\end{array}$ & 20.271 & $6 / 88$ & $\begin{array}{c}\text { HLADPB1, KLRC2, } \\
\text { KLRC4, IFNA21, KIR3DL2 }\end{array}$ & NFYB \\
\hline 4 & Adherens junction & 10.67 & $2 / 75$ & ACVR1B & PTPRB \\
\hline 5 & Type II diabetes mellitus & 7.677 & $2 / 44$ & $\begin{array}{l}\text { CACNA1C, } \\
\text { CACNA1E }\end{array}$ & \\
\hline 6 & Long term depression & 5.7 & $6 / 76$ & GRM1, NPR2, PRKCB1 & CRHR1, HRAS, PLA2G6 \\
\hline 7 & Gap junction & 5.297 & $8 / 96$ & GRM1, HTR2, NPR2, PRKCB1, TUBB6 & HRAS,PRKACB \\
\hline 8 & Tight junction & 5.253 & $10 / 135$ & CLDN22, CLDN6, MYH10, MYH6, PRKCB1 & $\begin{array}{c}\text { CLDN7, HRAS, MYH14, PP2R4, } \\
\text { PRKCQ }\end{array}$ \\
\hline 9 & Melanoma & 5.206 & $4 / 71$ & FGF11, FGF2, FGF18 & HRAS \\
\hline 10 & Basal cell carcinoma & 5.174 & $1 / 55$ & FZD2 & \\
\hline 11 & TGF beta signalling & 4.763 & $3 / 89$ & ACVR1B, DCN & ZFYVE9 \\
\hline$\overline{12}$ & Glioma & 4.756 & $4 / 64$ & PRKCB1 & CAMK2D, HRAS, SHC2 \\
\hline 13 & MAPK signalling pathway & 4.747 & $19 / 265$ & $\begin{array}{c}\text { CACNA1C, CACNA1E, CACNA1S, CACNB2, } \\
\text { ACVR1B, FGF11, FGF2, } \\
\text { FGF18, FOS, JUN, PAK1, PRKCB1 }\end{array}$ & $\begin{array}{c}\text { CRKL, HRAS, MAP3K7IP2, } \\
\text { PLA2G6, PRKACB, } \\
\text { PRKX }\end{array}$ \\
\hline
\end{tabular}

The 'primary 1.1 fold gene list' was loaded into Pathway Express to determine the significant epithelial pathways associated with co-culture with stroma in 3D. The 1.1 fold gene list was derived from all ten primary epithelial cell cultures. All significantly $(\mathrm{p}<0.05)$ ranked pathways are shown $(91$ ranked in total). 

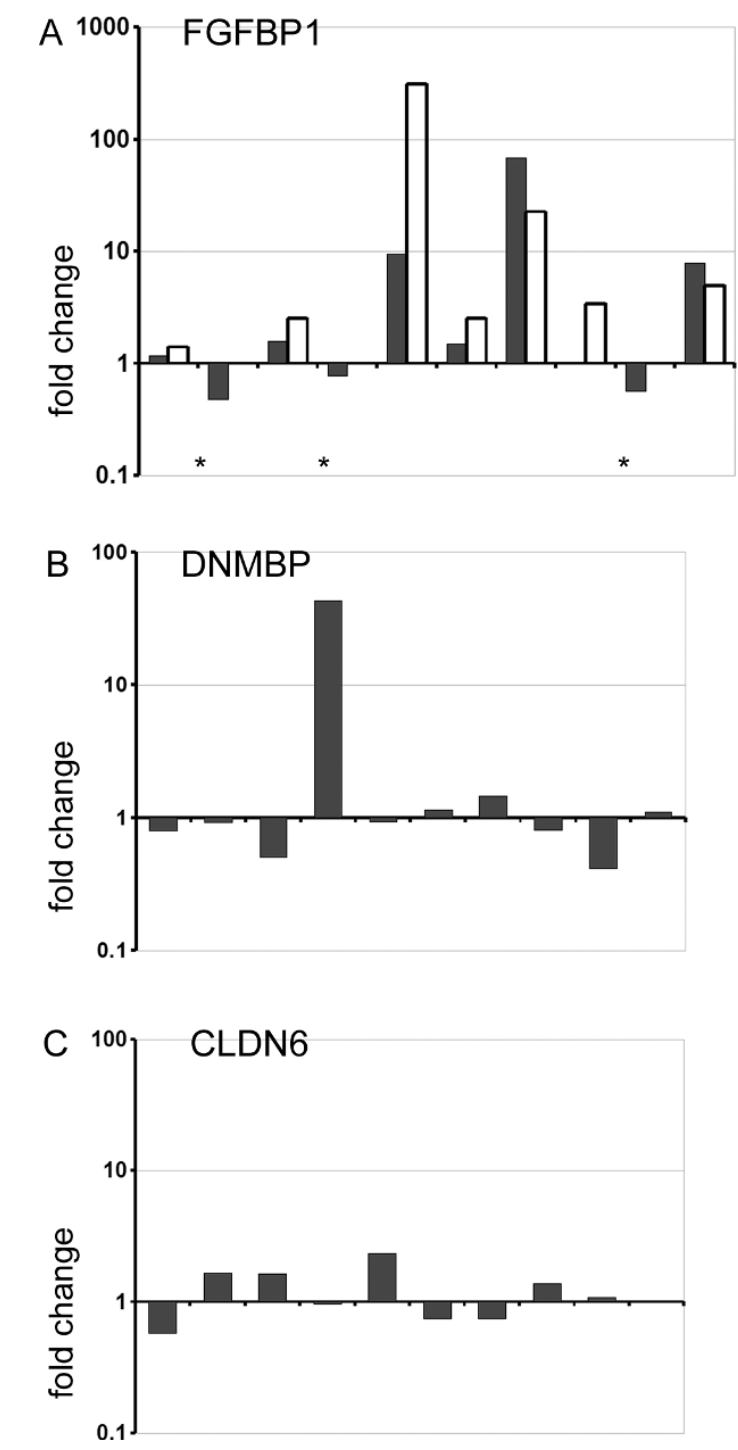

Figure 2 Evidence of heterogeneity in gene expression between different primary culture samples. Primary epithelial cells were grown for 14 days in Matrigel with stromal co-cultures and compared to cultures grown without stroma. RNA was prepared from single acini. Data is shown for all ten matched primary epithelial cell cultures. A) the mean fold change in gene expression levels for FGFBP1 measured by Operon microarray (black bars) or QRT-PCR (open bars). B) the mean fold change in gene expression levels for DNMBP measured by Operon microarray. C) the mean fold change in gene expression levels for CLDN6 measured by Operon microarray. Changes in gene expression detected by Operon were from the 1.1 fold gene list $(p<0.05)$.* insufficient sample available for QRT-PCR

verification of DNMBP expression (Figure 2B) and CLDN6 expression (Figure 2C) indicated that the culture/patient heterogeneity was not limited to FGFBP1. Although average gene expression of DNMBP and CLDN6 was upregulated (Tables 1 and 2), analysis of individual cultures/patient samples indicated that
DNMBP was upregulated in only $4 / 10$ samples and CLDN6 in 5/10 samples. It was evident that the mean fold change in expression was dependent predominantly on a low number of high differential expressors and was not typical of the whole population of epithelial samples.

\section{BPH-1 cell line gene expression changes and pathways} induced by stromal secreted factors in 3D culture

To overcome the problems of heterogeneity we decided to analyse a prostate epithelial cell line, BPH-1, which can also grow into acinus-like spheroids in 3D culture and demonstrates increased lateral adhesions, in response to stroma [10]. We performed a second microarray experiment to compare the RNA expression patterns between 3D BPH-1 acini grown with and without stromal co-culture. The cell line model array would then inform the primary culture model, allowing us to identify shared differentially expressed genes and pathways. This would provide a dataset that was relevant to human adult tissues, but within a reproducible cell line model. Common genes may also be more fundamental to adhesion and therefore of greater importance to future functional studies.

Three technical replicates of BPH-1 cells were cultured in 3D with and without primary stroma (an identical mix from three different patients), using identical culture conditions to the primary cell model. 7843 probe sets were differentially expressed between the two experimental groups $(\mathrm{p}<0.05)$. Table 3 lists the most differentially expressed genes and table 4 lists the pathways with an impact factor greater than 4 (62 pathways were ranked in total). The highest ranking pathway was ECM-receptor interactions. Eleven of the ranked pathways were significant and, of these, only TGF beta signalling was listed for both primary cells and cell lines datasets. KRT6B was highly down regulated in both models (table 1 and 3 ).

The TGF beta signalling pathway is significant for primary and $\mathrm{BPH}-1$ arrays

Figure 3 shows the Kyoto Encyclopedia of Genes and Genomes (Kegg) pathway for TGF beta signalling [15-17] and illustrates the significant genes found by Pathway Express for both primary and cell line microarray datasets. No gene was expressed by both arrays on the Kegg pathway. The primary cultures showed upregulation of ACVR1B (activin receptor type-1B) and DCN (decorin) and down regulation of SARA (ZFYVE9) in response to stromal co-culture. BPH-1 cells showed upregulation of INHBB (inhibin beta chain) and down regulation of FST (follistatin), MYC, THBS1 (thrombospondin 1) and ID1.

To verify the BPH-1 microarray data and in particular genes associated with TGF beta signalling pathway, we 
Table 3 The ten most upregulated and down regulated BPH-1 genes in response to stroma, in 3D culture

\begin{tabular}{|c|c|c|c|}
\hline Gene Symbol & Gene Title & $\begin{array}{c}\text { Fold } \\
\text { change }\end{array}$ & Probe ID \\
\hline MAP7D2 & MAP7 domain containing 2 & 2.866 & 228262_at \\
\hline IGFBP3 & insulin-like growth factor binding protein 3 & 2.613 & 212143_s_at \\
\hline IGFBP3 & insulin-like growth factor binding protein 3 & 2.600 & 210095_s_at \\
\hline STON1 & stonin 1 & 2.527 & 213413_at \\
\hline BEAN & brain expressed, associated with Nedd4 & 2.446 & 214068_at \\
\hline $\mathrm{FBXO32}$ & F-box protein 32 & 2.290 & 225803_at \\
\hline GNG7 & guanine nucleotide binding protein ( $G$ protein), gamma 7 & 2.277 & 228831_s_at \\
\hline $\mathrm{NOTCH} 3$ & Notch homolog 3 (Drosophila) & 2.264 & 203238_s_at \\
\hline $\mathrm{MB}$ & myoglobin & 2.258 & 204179_at \\
\hline CLIC3 & chloride intracellular channel 3 & 2.225 & 219529_at \\
\hline $\begin{array}{l}\text { KRT6A /// KRT6B /// } \\
\text { KRT6C }\end{array}$ & keratin $6 \mathrm{~A} / / /$ keratin $6 \mathrm{~B} / / /$ keratin $6 \mathrm{C}$ & -3.099 & 214580_x_at \\
\hline NNMT & nicotinamide $\mathrm{N}$-methyltransferase & -3.126 & 202237_at \\
\hline KIAA1199 & KIAA1199 & -3.183 & 212942_s_at \\
\hline KRT6A & keratin $6 \mathrm{~A}$ & -3.215 & 209125_at \\
\hline HSD17B2 & hydroxysteroid (17-beta) dehydrogenase 2 & -3.450 & 204818_at \\
\hline KRT14 & keratin 14 & -3.460 & 209351_at \\
\hline $\begin{array}{l}\text { SERPINB3 /// } \\
\text { SERPINB4 }\end{array}$ & $\begin{array}{l}\text { serpin peptidase inhibitor, clade B (ovalbumin), member } 3 \text { /// serpin peptidase inhibitor, clade B } \\
\text { (ovalbumin), member } 4\end{array}$ & -8.311 & 210413_x_at \\
\hline SERPINB3 & serpin peptidase inhibitor, clade $B$ (ovalbumin), member 3 & -9.406 & 209719_x_at \\
\hline SERPINB3 & serpin peptidase inhibitor, clade B (ovalbumin), member 3 & -12.240 & 209720_s_at \\
\hline SERPINB4 & serpin peptidase inhibitor, clade B (ovalbumin), member 4 & -17.045 & 211906_s_at \\
\hline
\end{tabular}

*Values are mean fold expression changes of three BPH-1 cultures abstracted from Affymetrix datasets. All genes showed significant differential expression ( $\mathrm{p}<$ 0.05). Positive values indicate upregulation, negative values indicate down regulated genes in the epithelial cells. Hypothetical or unassigned genes were removed from the list.

used a commercial PCR array for the 'human TGF beta/ BMP signaling pathway'. The differential expression of fourteen genes was verified (Figure 4); BGLAP (osteocalcin), bone morphogenic proteins and receptors (BMP, BMPR), type 1 collagens (COL1), TGF beta induced (TGFBI) and TGF beta receptors 2 and 3, IGFBP3 (Insulin-like growth factor binding protein 3), PLAU (Urokinase-type plasminogen activator), FKBP1B (peptidyl-prolyl cis-trans isomerase), SOX4 and EVI1 (Ecotropic viral integration site 1). THBSP1, ACVR1B, DCN and ZFYVE9 did not appear on the QRT-PCR. Twenty one differentially expressed genes in the microarray data were not confirmed by QRT-PCR (results not shown). The low correlation between the microarray data and QRT-PCR using the same cell line is likely due to stromal heterogeneity (the experiments were performed with different stromal culture mixes). However, the genes in agreement will represent more robust candidates

Identification of common genes which are upregulated or down regulated in both primary and $\mathrm{BPH}-1$ microarray datasets

To aid the identification of genes that are most relevant to human adult tissue we directly compared the gene lists from the microarray analysis of primary cells to those from the microarray analysis of cell line, this identified 36 genes which were upregulated in both lists and 45 genes that were down regulated (Figure 5). Interestingly, only three genes from tables 2 and 4 describing the highly differentially expressed genes in either model appeared in this figure (KRT6B, TOP1 and GNG7) and none of these genes have a known function relating to morphology. To identify genes most likely to have a function in morphology or adhesion, the gene ontology (GO) molecular function and cellular component terms were found for each gene and then we identified all genes which contained the phrases 'TGF beta', 'E-cadherin', 'tight junctions', 'actin', 'cytoskeleton', 'cell shape', 'cell adhesion'. Several gene groups were identified: actin binding (GO:0003779), FHOD3, ABLIM1, TMOD4, MYH10; actin cytoskeleton organisation (GO: 0030036), DIAPH2, FHOD3; regulation of Rho signal transduction (GO:0035023), BCR; regulation of cell shape (GO:00 08360), MYH10; cell morphogenesis (GO:0000902), STK4; microtubule (GO:0005874), MAP2, KIFC1; cellmatrix adhesion/cell adhesion (GO:0007160, GO:0007155), NID2, CD44, ITGA6. In addition we identified a large group of genes associated with ion channel/ion transporter activity (GO:0005244, 
Table 4 Significant pathways associated with the response of BPH-1 cells to stroma in 3D culture

\begin{tabular}{|c|c|c|c|c|c|}
\hline Rank & Pathway Name & $\begin{array}{l}\text { Impact } \\
\text { Factor }\end{array}$ & $\begin{array}{l}\text { no. genes/ genes in } \\
\text { pathway }\end{array}$ & Upregulated genes & Downregulated genes \\
\hline 1 & ECM-receptor interaction & $14.5^{*}$ & $10 / 84$ & COL6A1, LAMB2 & CD44, CD47, FN1, ITGA2, LAMB1, THBS1 \\
\hline 2 & Adherens junction & 11.94 & $1 / 78$ & & MET \\
\hline 3 & Haematopoietic cell lineage & $9.93^{*}$ & $8 / 87$ & & $\begin{array}{c}\text { CD44, CD55. CD59, IL1R2, IL4R, IL7R, } \\
\text { ITGA2, ITGA6 }\end{array}$ \\
\hline 4 & Small cell lung cancer & $7.96^{*}$ & $7 / 86$ & LAMB2 & FN1, ITGA2, ITGA6, LAMB1, MYC \\
\hline 5 & Basal cell carcinoma & 7.83 & $2 / 55$ & WNT10A, WNT4 & \\
\hline 6 & Pathways in cancer & $7.27^{*}$ & $13 / 330$ & $\begin{array}{l}\text { DAPK2, LAMB2, WNT10A, } \\
\text { WNT4 }\end{array}$ & $\begin{array}{c}\text { ETS1, FN1, ITGA2, ITGA6, LAMB1, MET, } \\
\text { MYC, RUNX1 }\end{array}$ \\
\hline 7 & Focal adhesion & $7.02^{*}$ & $10 / 203$ & COL6A1, LAMB2 & FLNB, FN1, ITGA2, LAMB1, MET, THBS1 \\
\hline 8 & TGF-beta signalling pathway & $6.61^{*}$ & $5 / 87$ & INHBB & FST, MYC, THBS1, ID1 \\
\hline 9 & Axon guidance & $6.48^{*}$ & $7 / 129$ & $\begin{array}{l}\text { EFNB3, NFATC4, NTN1, } \\
\text { SEMA3C }\end{array}$ & MET, PLXNC1, SRGAP1 \\
\hline 10 & Jak-STAT signalling pathway & $6.32^{*}$ & $7 / 155$ & & $\begin{array}{c}\text { IL13RA2, IL24, IL4R, IL7R, MYC, OSMR, } \\
\text { SOCS3 }\end{array}$ \\
\hline 11 & Calcium signalling pathway & 4.93 & $2 / 182$ & & EDNRA, HRH1 \\
\hline 12 & Bladder cancer & $4.74^{*}$ & $3 / 42$ & DAPK2 & MYC, THBS1 \\
\hline 13 & $\begin{array}{l}\text { B cell receptor signalling } \\
\text { pathway }\end{array}$ & 4.62 & $2 / 65$ & BLNK, NFATC4 & \\
\hline 14 & $\begin{array}{l}\text { Complement and coagulation } \\
\text { cascades }\end{array}$ & $4.59^{*}$ & $4 / 69$ & $\mathrm{C} 3$ & CD55, CD59, F3 \\
\hline 15 & $\begin{array}{l}\text { Cytokine-cytokine receptor } \\
\text { interaction }\end{array}$ & $4.56^{*}$ & $9 / 263$ & INHBB & $\begin{array}{c}\text { CXCL5, IL1R2, IL24, IL4R, IL7R, MET, } \\
\text { OSMR, PPBPP }\end{array}$ \\
\hline 16 & $\begin{array}{l}\text { Regulation of actin } \\
\text { cytoskeleton }\end{array}$ & 4.37 & $5 / 217$ & CYFIP2 & FN1, ITGA2, ITGA6, SSH1 \\
\hline 17 & Regulation of autophagy & 4.32 & $1 / 35$ & GABARAPL1 & \\
\hline 18 & Melanoma & 4.25 & $1 / 71$ & & MET \\
\hline 19 & Wnt signaling pathway & 4.2 & $4 / 152$ & NFATC4, WNT10A, WNT4 & MYC \\
\hline 20 & Tight junction & 4.11 & $2 / 135$ & JAM2, TJP3 & \\
\hline
\end{tabular}

The 'BPH1 1.1 fold gene list' was loaded into pathway express. All pathways with an impact factor $>4$ are listed. ${ }^{*}$ significant pathways, $\mathrm{p}<0.05$. The 1.1 fold gene list was derived from all three $\mathrm{BPH}-1$ cultures.

GO:0005216, GO:0015081, GO:0046873), CACNA1C, CACNB2, KCNH2, SLC8A1, SLC39A9. The remaining genes were predominantly associated with transcription, metabolism or protein transport. We further identified genes associated with developmental signalling pathways, using GO terms or literature searches, this identified; POFUT1, (notch signalling); IRX2, HOXA5 (homeobox genes); FZD2 (Wnt signalling); FGF11, SOX4 (TGF beta) and SMARCC1. All these developmental pathways have known and complex roles in prostate development or in the remodelling of epithelial sheets, their function within our model remains to be determined $[18,19]$. Importantly SOX4 is associated with TGF beta signalling [20] though it was not listed within the Kegg pathway. Stromal function was confirmed by the down regulation of CD44, ITGA6 and KRT6, downregulation of these genes is associated with epithelial differentiation, a known role of stroma [21,22]. MAP2 was chosen to validate the list of common genes. Using QRT-PCR we confirmed that BPH-1 cells cultured in the presence of stroma (different stromal mix to either array) had upregulated MAP2 expression (Figure 6).

\section{Discussion}

This research highlights the difficulties faced by a cell biologist trying to select the most appropriate model system for their research. In our work we prefer to validate all our experiments using primary cultures to ensure our research reflects human biology and disease. The use of a single cell line for experiments is common because they provide a reliable and repeatable model. However cell lines often suffer from 'genetic drift' in long term culture and do not reflect the tissue from which they were derived nor their original architecture and can often provide inadequate data [23]. Experimentation on a panel of cell lines should be adopted to demonstrate that a result holds true across many models and not just one particular laboratory model. However, as demonstrated here, the use of a wider range of cell models reduces our ability to find valid genes from a 


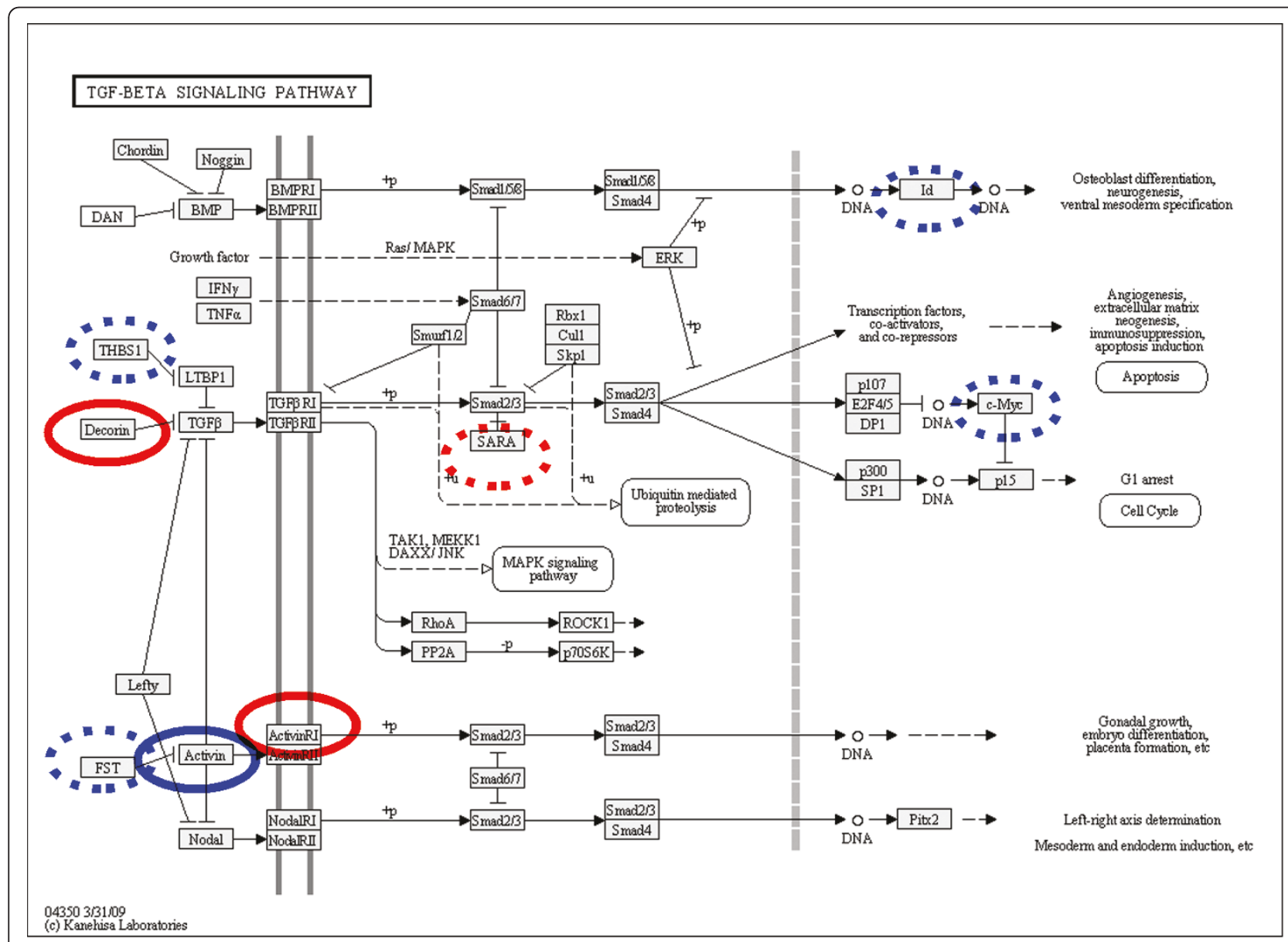

Figure 3 Genes identified in the TGF beta pathway. The Kegg pathway for TGF beta signalling illustrating the significant genes identified by pathway express. Genes circled in red indicate genes indicate genes from the primary cell microarray dataset (Operon) and those in blue from the $\mathrm{BPH}-1$ cell microarray dataset (Affymetrix). Continuous lines are upregulated genes whereas broken lines are down-regulated.

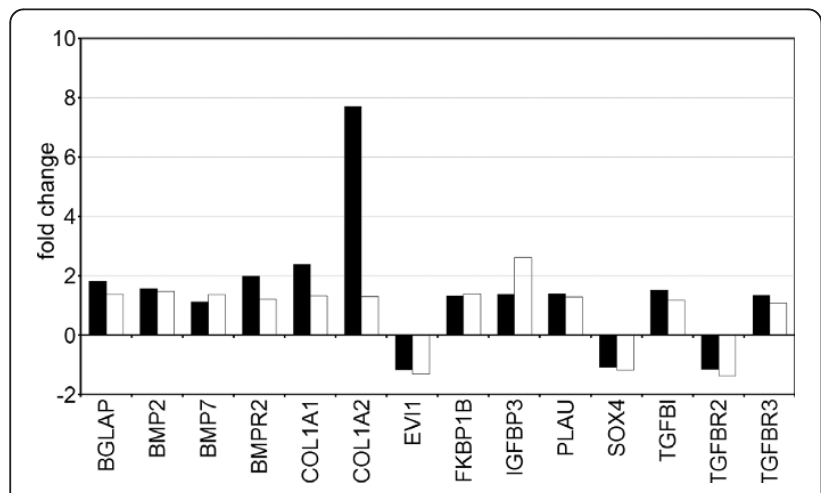

Figure 4 Validation of human TGF beta/ BMP signalling pathway genes. Average changes in gene expression between 3D $\mathrm{BPH}-1$ acini cultured with stroma compared to without stroma detected by Affymetrix (white bars) and QRT-PCR techniques (black bars). Changes in gene expression detected by Affymetrix were from the 1.1 fold gene list $(p<0.05)$. microarray analysis. Indeed, multiple testing correction was not applied to the primary microarray because this produced no significant genes, due to the large heterogeneity between patient samples of primary epithelia and stromal cells. Such heterogeneity is common place when working with human tissues $[24,25]$. The problems produced by heterogeneity can be reduced by increasing the number of samples. Primary cultures are technically difficult to produce and take time to acquire. Rather than increase our sample size, which was already considerable, we decided to compare our data to that of a cell line model and combine several stromal cultures into one (to reduce the heterogeneity from the stroma). By combining the two microarray analyses and analysing common pathways as well as individual genes, we hope to identify tissue relevant genes in a cell line. These genes may also have more fundamentally importance to the mechanism of increased adhesion. Future work will 


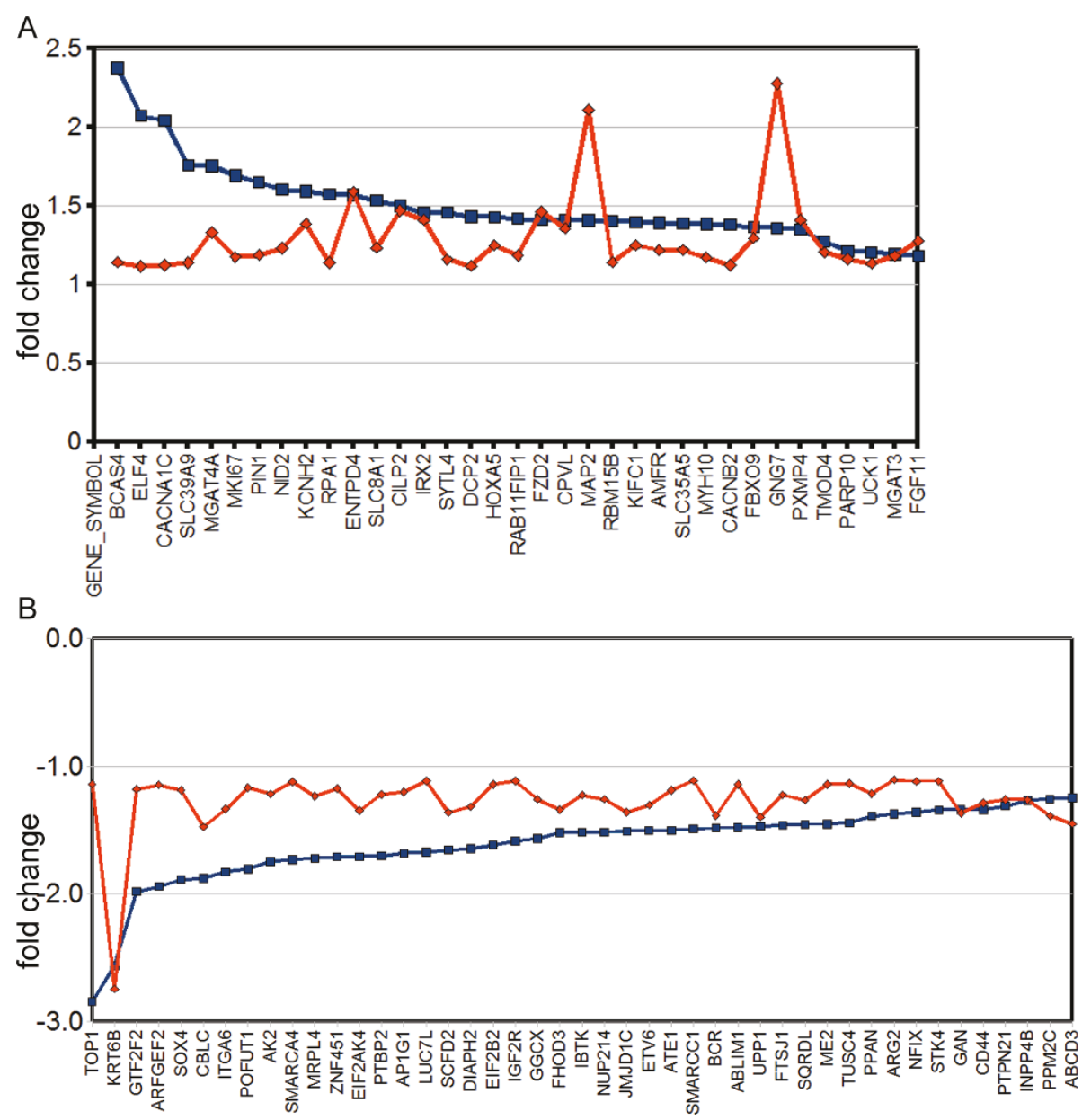

Figure 5 Identification of common genes that were up regulated or down regulated in both cell line and primary epithelial models in response to stroma. Fold change lists were generated using Genesping (both used fold change lists of 1.1 fold and $p<0.05$ ) for both the primary (blue) and cell line (red) arrays. The gene lists were compared using Entrez gene IDs to find common upregulated (A) and downregulated genes (B).

seek to provide functional confirmation of the identified genes and pathways and confirm that the genes have the same function within the cell line and the primary models and whether this relates to normal tissue. At this point the work remains preliminary until future functional studies are carried out.

Using 3D cultures to model the stromal maintenance of adult epithelial tissues, we previously discovered that stromal cells signal to increase the lateral cell adhesions of epithelial cells [7]. This is an intriguing discovery since in monolayer epithelial cells are well known to scatter in response to stroma or stromal conditioned media [26]. Examination of the genes that were highly upregulated or highly down regulated during this morphological change by two arrays of primary and cell line models showed little agreement. Only KRT6 was highly down regulated in both. Examination of the significant pathways identified only TGF beta signalling, and further examination of the genes within the pathway identified only one, SOX4, to have common expression. One interpretation of these results is that there is poor agreement between the models and this is certainly true but the genes identified in common are likely to be more relevant and more fundamental to the processes under study. Analysis of common genes identified important morphological clustering of genes, with the following terms; actin binding, cytoskeleton, ion channel/ion transporter activity and genes associated with developmental pathways. The present knowledge of these genes with particular reference to morphology will be discussed.

The importance of TGF beta signalling has confirmed our earlier observation that stromal derived TGF beta is important for the control of lateral epithelial cell adhesions (paper in submission). SOX4 is an important transcription factor in development and interacts with many 


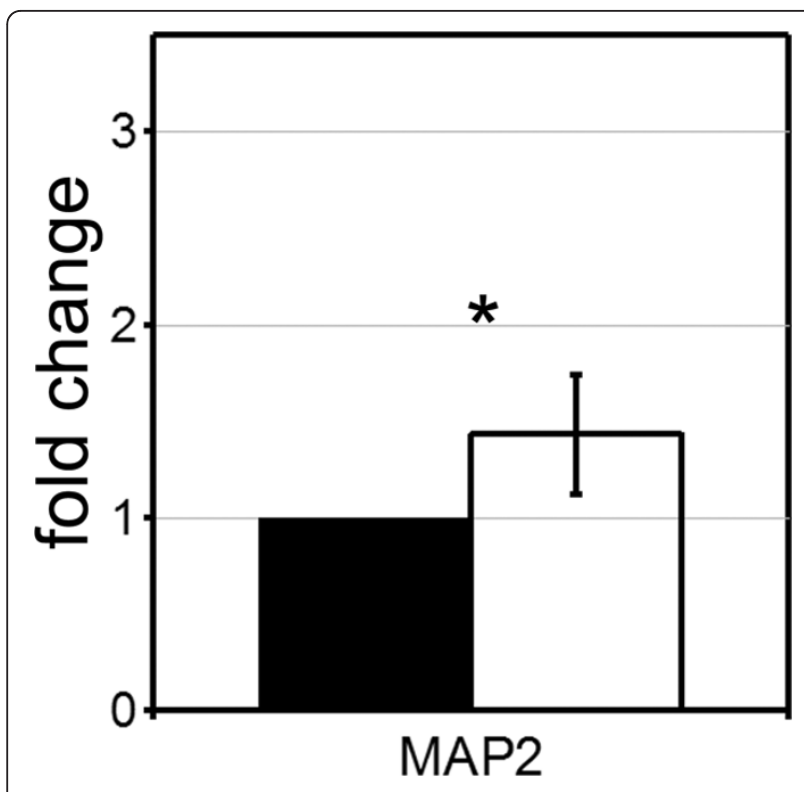

Figure 6 Confirmation of MAP2 expression. QRT-PCR was performed on mRNA isolated from 3D BPH-1 acini cultured for 7 days with (white) or without stroma (black) from three different patients (in duplicate). The average fold difference in gene expression was expressed relative to growth without stroma \pm standard error ** $\mathrm{P}<0.05$

morphology related pathways, (Hedgehog, Notch and Wnt). SOX4 stabilises $\beta$-catenin protein and enhances $\beta$-catenin/TCF activity [20]. Over-expression of SOX4 is associated with many cancers (including prostate) and anchorage independent growth [27]. The association of increased adhesion with down-regulation of SOX4, found here, is an important mechanism to study further. Other genes associated with TGF beta signalling were identified from the list of common genes, these were STK4, ITGA6 and CILP2. STK4 is an important signal transducer for the TGF beta family [28]. TGF beta induced down regulation of ITGA6 and upregulation of CILP2 (cartilage intermediate layer protein 2) has already been demonstrated in other tissue models $[29,30]$. Therefore these genes may provide good candidates with which to test the importance of TGF beta signalling in our model and also the importance of stromal derived TGF beta.

Actin binding and cytoskeleton genes provided the most likely set of genes to have a role in adhesion. We found up regulation of MAP2 (microtubule-associated protein 2), which is a major regulator of microtubule dynamics and is best known for its role in neuronal development [31]. MAP2 is also located in non-neuronal cells where its biological functions remain largely unknown, it can interact with F-actin and increase cellular migration by inducing microtubule bundling at the cell periphery [32]. Microtubules are known to contact adherens junctions and are required (alongside myosin II) for cadherin junction formation [33]. Microtubules may prove to be an important area for future focus due the further identification of KIFC1, a kinesin/microtubule motor protein with important functions in polarity and cell division [34]. Several actin associated genes were found including DIAPH2 (Diaphanous 2), FHOD3 (formin homology 2 domain 3), BCR (breakpoint cluster region), ABLIM1 (actin binding LIM protein 1), MYH10 (non-muscle myosin heavy chain IIB or NMII) and TMOD4 (tropomodulin 4). Diaphanous proteins localise to cell to cell contacts where they also play an important role in cadherin junction formation [35-37]. Formin homology 2 sequences are essential to induce actin assembly, but also inhibit actin elongation [38]. BCR can regulate the activity of Rho-like GTPases and is thought to regulate signalling pathways at the sites of cellular junctions $[39,40]$. ABLIM1 has uncertain biological function, but it may act as a scaffold protein [41]. MYH10 has a fundamental role in processes that require cellular reshaping and movement. NMII uses actin cross-linking and contractile functions to regulate the actin cytoskeleton. It has complex roles in migration, polarity and the formation and promotion of stable cell-cell junctions [42]. Crucially, NM II-driven mechanisms also govern the three dimensional organization of epithelial tissues, studied in X. laevis and D. melanogaster during early embryonic development and organogenesis. Therefore upregulation of MY10 found here may promote polarity and adhesion. TMOD4 is an actin filament capping protein that maintains the length of the actin filaments in skeletal muscle and in has a role in cell membrane dynamics $[43,44]$. None of these cytoskeletal genes have known functions associated with the prostate.

Several calcium and potassium channels were up regulated on both arrays (CACNA1C, CACNB2, KCNH2, SLC8A1) these may provide a means of modulating cell junctions by controlling the intracellular levels of calcium and potassium [45]. Recent bioinformatic and proteomic analysis of epithelial tight junctions revealed that synaptic proteins and signalling molecules were associated with tight junctions, and these included potassium and calcium voltage gated channels [46]. The authors suggested that tight junctions may have a novel role as an epithelial synapse for cell to cell communication. Validation of these results may provide further insights into this hypothesis.

Before this study, tight junctions and adherens junctions were likely candidates to be involved in increased cell to cell adhesion. They are dynamic structures linked to the acto-myosin cytoskeleton and are regulated by Rho/Ras-GTPases [19]. Microarray analysis did not indicate a clear role for either junction. Junctional pathways and genes were associated with either primary or cell 
line model but not both. Since adhesion depends on the interaction of junctional proteins with the cytoskeleton, our combined analysis indicates a greater role for the cytoskeleton and its regulators over that of junctional proteins in adhesion. This is an important finding which may have been missed without combining the models and will be important to prove further. Significantly MYH10 can regulate the assembly of apical junction complexes and increase the height of lateral cell domains [47], its inhibition reduces tight junctions and adherens junction formation [47-50]. Both tight junctions and adherens junctions are controlled by the TGF beta superfamily. The effect of TGF beta on adhesion varies according to the experimental model, making a strict interpretation difficult. TGF beta can antagonise tight junction formation in cell lines [51,52] but increase barrier function through upregulation of claudins in tissue [53]. Although TGF beta is known to initiate epithelial-mesenchymal transition it can also promote adhesion by targeting E-cadherin to the cell membrane via the ELF adaptor protein (ELF4 was highly upregulated by both arrays) [54].

\section{Conclusions}

We used microarray analysis and bioinformatics to identify candidate epithelial genes which control lateral cell adhesion under stromal stimulation. We confirmed the importance of TGF beta signalling, and in particular SOX4. Analysis of genes that were common to both cell line and primary arrays found several morphology related gene clusters; actin binding, GTPase activator activity, cytoskeleton, protein binding, proteinaceous extracellular matrix, ion channel/ion transporter activity and genes associated with developmental pathways. These candidates will be investigated in future functional studies. This work highlights the complexity of any biological process and the value of combining gene array data from different models to identify important pathways and genes. Overall we have shown the complexity of stromal controlled epithelial morphology. The study of intercellular adhesion is a fast expanding field, and our identification of genes associated with actin binding, microtubules and anion signalling complements newly emerging ideas.

\section{Additional material}

Additional file 1: Table S1: Primers and probes for QRT-PCR prime sequences.

\section{Acknowledgements and Funding}

We thank Prof. Norman Maitland for his generous support of the project and provision of laboratory space. Many thanks to M. Stower for providing prostate tissue samples and to Simon Hayward for providing the $\mathrm{BPH}-1$ cell lines. We also thank the members of the Yorkshire Cancer Research laboratory for their useful discussions and technical help. This work was supported by the Wellcome Trust [GR076612MA] and Biotechnology and Biological Sciences Research Council, UK [BB/E021409/1]. The funders had no role in study design, data collection and analysis, decision to publish, or preparation of the manuscript.

\section{Author details}

${ }^{1}$ Yorkshire Cancer Research Unit, Dept. Biology, University of York, Heslington, York, (YO10 5YW), UK. ² Genomics Laboratory, Technology Facility, Dept. Biology, University of York, Heslington, York, (YO10 5YW), UK. ${ }^{3}$ Division of Oncogenomics, Department of Pathology, University of Regensburg, Regensburg, Germany.

\section{Authors' contributions}

KC participated in the design of the study, performed data analysis and design, prepared the primary culture samples and coordinated the Operon array study. JFP prepared samples for Affymetrix array and performed qRTPCR assays. MG and CK coordinated and performed the Operon array. NA coordinated the Affymetrix array and was involved in data analysis. DP was involved in data analysis. SHL conceived of the study, participated in study design and co-ordination, analysed experiments and wrote the manuscript. All authors read and approved the final manuscript.

\section{Competing interests}

The authors declare that they have no competing interests.

Received: 4 February 2011 Accepted: 22 June 2011

Published: 22 June 2011

\section{References}

1. Lecuit T, Lenne PF: Cell surface mechanics and the control of cell shape, tissue patterns and morphogenesis. Nature Rev Mol Cell Biol 2007, 8:633-644.

2. Halbleib JM, Nelson WJ: Cadherins in development: cell adhesion, sorting, and tissue morphogenesis. Genes \& Development 2008, 20:3199-3214

3. Kemler R: From cadherins to catenins: cytoplasmic protein interactions and regulation of cell adhesion. Trends in Genetics 1993, 9:317-321.

4. Cavey $M$, Rauzi $M$, Lenne $P$, Lecuit $T$ : A two-tiered mechanism for stabilization and immobilization of E-cadherin. Nature 2008, 453:751-756.

5. Cereijido M, Contreras RG, Shoshani L: Cell Adhesion, Polarity, and Epithelia in the Dawn of Metazoans. Physiol Rev 2004, 84:1229-1262.

6. Balda MS, Matter K: Tight junctions and the regulation of gene expression. Biochim Biophys Acta 2009, 1788:761-7.

7. Lang SH, Stark M, Collins A, Paul A, Stower MJ, Maitland NJ: Experimental Prostate Epithelial Morphogenesis in Response to Stroma and ThreeDimensional Matrigel Culture. Cell Growth \& Differentiation 2001, 12:631-640.

8. Thiery JP, Sleeman J: Complex networks orchestrate epithelialmesenchymal transitions. Nat Rev Mol Cell Biol 2006, 7:131-142.

9. Hayward SW, Del Buono R, Deshpande N, Hall PA: A functional model of adult human prostate epithelium. The role of androgens and stroma in architectural organisation and the maintenance of differentiated secretory function. J Cell Sci 1992, 102:361-372.

10. Pearson JF, Hughes S, Chambers K, Lang SH: Polarised fluid movement and not cell death, creates luminal spaces in adult prostate epithelium. Cell Death Differentiation 2009, 16:475-482.

11. Hartmann $\mathrm{CH}$, Klein CA: Gene expression profiling of single cells on large-scale oligonucleotide arrays. Nucleic Acids Res 2006, 34:e143.

12. Li C, Wong WH: Model-based analysis of oligonucleotide arrays: Expression index computation and outlier detection. PNAS 2001, 98:31-36.

13. Chambers KF, Bacon JR, Kemsley EK, Mills RD, Ball RY, et al: Gene expression profile of primary prostate epithelial and stromal cells in response to sulforaphane or iberin exposure. Prostate 2009, 69:1411-1421.

14. Draghici S, Khatri P, Tarca AL, Amin K, Done A, et al: A systems biology approach for pathway level analysis. Genome Research 2007, 17:1537-1545.

15. Kanehisa M, Goto S, Furumichi M, Tanabe M, Hirakawa M: KEGG for representation and analysis of molecular networks involving diseases and drugs. Nucleic Acids Res 2010, 38:D355-D360. 
16. Kanehisa M, Goto S, Hattori M, Aoki-Kinoshita KF, Itoh M, et al: From genomics to chemical genomics: new developments in KEGG. Nucleic Acids Res 2006, 34:D354-357.

17. Kanehisa M, Goto S: KEGG: Kyoto Encyclopedia of Genes and Genomes. Nucleic Acids Res 2000, 28:27-30

18. Prins GS, Putz O: Molecular signaling pathways that regulate prostate gland development. Differentiation 2008, 76:641-59.

19. Jamora C, Fuchs E: Intercellular adhesion, signalling and the cytoskeleton. Nature Cell Biology 2002, 4:101-108.

20. Sinner D, Kordich JJ, Spence JR, Opoka R, Rankin S, et al: Sox17 and Sox4 Differentially Regulate \{beta\}-Catenin/T-Cell Factor Activity and Proliferation of Colon Carcinoma Cells. Mol Cell Biol 2007, 27:7802-7815.

21. Liu AY, True LD, LaTray L, Nelson PS, Ellis WJ, et al: Cell-cell interaction in prostate gene regulation and cytodifferentiation. Proc Natl Acad Sci USA 1997, 94:10705-10710.

22. Schmelz M, Moll R, Hesse U, Prasad AR, Gandolfi JA, et al: Identification of a stem cell candidate in the normal human prostate gland. Eur I Cell Biol 2005, 84:341-354.

23. Love HD, Booton SE, Boone BE, Breyer JP, Koyama T, et al: Androgen Regulated Genes in Human Prostate Xenografts in Mice: Relation to BPH and Prostate Cancer. PLOS ONE 2009, 4(12):e8384

24. Rose $A, X u Y$, Chen Z, Fan Z, Stamey TA, et al: Comparative gene and protein expression in primary cultures of epithelial cells from benign prostatic hyperplasia and prostate cancer. Cancer Lett 2005, 227:213-22.

25. Ahmed AA, Brenton JD: Microarrays and breast cancer clinical studies: forgetting what we have not yet learnt. Breast Cancer Research 2005, 7:96-99.

26. Chambers KF, Pearson JF, Aziz N, OToole P, Garrod D, Lang SH: Stroma Regulates Increased Epithelial Lateral Cell Adhesion in 3D Culture: A Role for Actin/Cadherin Dynamics. PLOS ONE 2011, 6(4):e18796.

27. Moreno CS: The Sex-Determining Region Y-Box 4 and Homeobox C6 Transcriptional Networks in Prostate Cancer Progression: Crosstalk with the Wnt, Notch, and PI3K Pathways. Am J Pathol 2005, 176:518-527.

28. Josso N, di Clemente N: Serine/threonine kinase receptors and ligands. Current Opinion in Genetics \& Development 1997, 7:371-377.

29. Lim JM, Kim JA, Lee JH, Joo CK: Downregulated expression of integrin alpha6 by transforming growth factor-beta(1) on lens epithelial cells in vitro. Biochem Biophys Res Commun 2001, 284:33-41.

30. Mori M, Nakajima M, Mikami Y, Seki S, Takigawa M, et al: Transcriptional regulation of the cartilage intermediate layer protein (CILP) gene. Biochemical and Biophysical Research Communications 2006, 341:121-127.

31. Dehmelt L, Halpain S: The MAP2/Tau family of microtubule-associated proteins. Genome Biology 2004, 6:204.

32. Liu SY, Chen YT, Tseng MY, Hung CC, Chiang WF, et al: Involvement of microtubule-associated protein 2 (MAP2) in oral cancer cell motility: a novel biological function of MAP2 in non-neuronal cells. Biochem Biophys Res Commun 2008, 366:520-5.

33. Stehbens SJ, Paterson AD, Crampton MS, Shewan AM, Ferguson C, et al: Dynamic microtubules regulate the local concentration of E-cadherin at cell-cell contacts. J Cell Sci 2006, 119:1801-1811.

34. Vale RD: The molecular motor toolbox for intracellular transport. Cell 2003, 112:467-480

35. Ryu JR, Echarri A, Li R, Pendergast AM: Regulation of Cell-Cell Adhesion by Abi/Diaphanous Complexes. Mol Cell Biol 2009, 29:1735-1748.

36. Faix J, Grosse R: Staying in Shape with Formins. Developmental Cell 2006, 10:693-706.

37. Beli P, Mascheroni D, Xu D, Innocenti M: WAVE and Arp2/3 jointly inhibit filopodium formation by entering into a complex with mDia2. Nature Cell Biology 2008, 10:849-857.

38. Paul AS, Pollard TD: Review of the Mechanism of Processive Actin Filament Elongation by Formins. Cell Motility and the Cytoskeleton 2009, 66:606-617.

39. Radziwill G, Erdmann RA, Margelisch U, Moelling K: The Bcr Kinase Downregulates Ras Signaling by Phosphorylating AF- 6 and Binding to Its PDZ Domain. Mol Cell Biol 2003, 23:4663-4672.

40. Zheng $X$, Güller S, Beissert T, Puccetti E, Ruthardt M: BCR and its mutants, the reciprocal $t(9 ; 22)$-associated $A B L / B C R$ fusion proteins, differentially regulate the cytoskeleton and cell motility. BMC Cancer 2006, 6:262.

41. Roof DJ, Hayes A, Adamian M, Chishti AH, Li T: Molecular Characterization of abLIM, a Novel Actin-binding and Double Zinc Finger Protein. Journal of Cell Biol 1997, 138:575-588.
42. Vicente-Manzanares JA, Ma M, Adelstein X, Horwitz RS, Rick A: Non-muscle myosin II takes centre stage in cell adhesion and migration. Nat Rev Mol Cell Biol 2009, 10:778-790.

43. Almenar-Queralt A, Lee A, Conley CA, Ribas de Pouplana L, Fowler VM: Identification of a novel tropomodulin isoform, skeletal tropomodulin, that caps actin filament pointed ends in fast skeletal muscle. J Biol Chem 1999, 274:28466-75.

44. Kostyukova AS, Hitchcock-DeGregori S, Greenfield NJ: Molecular Basis of Tropomyosin Binding to Tropomodulin, an Actin-capping Protein. J Mol Biol 2007, 372:608-618

45. Brown RC, Davis TP: Calcium Modulation of Adherens and Tight Junction Function: A Potential Mechanism for Blood-Brain Barrier Disruption After Stroke. Stroke 2002, 33:1706-1711

46. Tang W: Proteomic and bioinformatic analysis of epithelial tight junction reveals an unexpected cluster of synaptic molecules. Biology Direct 2006, 1:37.

47. Zhang J, Betson M, Erasmus J, Zeikos K, Bailly M, et al: Actin at cell-cell junctions is composed of two dynamic and functional populations. J Cell Sci 2005, 118:5549-5562.

48. Miyakea $Y$, Inouea N, Nishimurab K, Kinoshitaa N, Hosoyac H, Yonemura S: Actomyosin tension is required for correct recruitment of adherens junction components and zonula occludens formation. Exp Cell Res 2006, 312:1637-50.

49. Ivanov Al, Bachar M, Babbin BA, Adelstein RS, Nusrat A, et al: A Unique Role for Nonmuscle Myosin Heavy Chain IIA in Regulation of Epithelial Apical Junctions. PLOS ONE 2007, 2(8):e658.

50. Choi CK, Vicente-Manzanares M, Zareno J, Whitmore LA, Mogilner A Horwitz AR: Actin and alpha actinin orchestrate the assembly and maturation of nascent adhesions in a myosin II motor independent manner. Nature Cell Biology 2008, 10:1039-50.

51. Woo PL, Cha HH, Singer KL, Firestone GL: Antagonistic Regulation of Tight Junction Dynamics by Glucocorticoids and Transforming Growth Factorin Mouse Mammary Epithelial Cells. J Biol Chemistry 1996, 271:404-412.

52. Ozdamar B, Bose R, Barrios-Rodiles M, Wang H, Zhang Y, Wrana JL: Regulation of the Polarity Protein Par6 by TGF $\beta$ Receptors Controls Epithelial Cell Plasticity. Science 2005, 307:1603-1608.

53. Howe KL, Reardon C, Wang A, Nazli A, McKay DM: Transforming growth factor-beta regulation of epithelial tight junction proteins enhances barrier function and blocks enterohemorrhagic Escherichia coli 0157:H7induced increased permeability. Am J Pathol 2005, 167:1587-1597.

54. Katuri $V$, Tang Y, Li C, Jogunoori W, Deng CX, et al: Critical interactions between TGF-beta signaling/ELF, and E-cadherin/beta-catenin mediated tumor suppression. Oncogene 2006, 25:1871-86.

doi:10.1186/1423-0127-18-45

Cite this article as: Chambers et al:: Stromal upregulation of lateral epithelial adhesions: Gene expression analysis of signalling pathways in prostate epithelium. Journal of Biomedical Science 2011 18:45.

\section{Submit your next manuscript to BioMed Central and take full advantage of:}

- Convenient online submission

- Thorough peer review

- No space constraints or color figure charges

- Immediate publication on acceptance

- Inclusion in PubMed, CAS, Scopus and Google Scholar

- Research which is freely available for redistribution

Submit your manuscript at www.biomedcentral.com/submit
C Biomed Central 